\title{
Effects of Solvents, Emulsions, Cosolvents, and Complexions on Ex Vivo Mouse Myometrial Contractility
}

\author{
Christopher J. Hansen ${ }^{1}$ - Shajila Siricilla ${ }^{1}$. Naoko Boatwright ${ }^{1}$. Jackson H. Rogers ${ }^{1}$. Melissa E. Kumi ${ }^{1}$. \\ Jennifer Herington ${ }^{1,2}$ (D)
}

Received: 16 February 2021 / Accepted: 2 April 2021 / Published online: 14 April 2021

(C) The Author(s) 2021

\begin{abstract}
A great need exists to develop tocolytic and uterotonic drugs that combat poor, labor-related maternal and fetal outcomes. A widely utilized method to assess novel compounds for their tocolytic and uterotonic efficacy is the isometric organ bath contractility assay. Unfortunately, water-insoluble compounds can be difficult to test using the physiological, buffer-based, organ bath assay. Common methods for overcoming solubility issues include solvent variation, cosolvency, surfactant or complexion use, and emulsification. However, these options for drug delivery or formulation can impact tissue function. Therefore, the goal of this study was to evaluate the ability of common solvents, surfactants, cosolvents, and emulsions to adequately solubilize compounds in the organ bath assay without affecting mouse myometrial contractility. We found that acetone, acetonitrile, and ethanol had the least effect, while dimethylacetamide, ethyl acetate, and isopropanol displayed the greatest inhibition of myometrial contractility based on area under the contractile curve analyses. The minimum concentration of surfactants, cosolvents, and human serum albumin required to solubilize nifedipine, a current tocolytic drug, resulted in extensive bubbling in the organ bath assay, precluding their use. Finally, we report that an oil-in-water base emulsion containing no drug has no statistical effect beyond the control (water), while the drug emulsion yielded the same potency and efficacy as the freely solubilized drug.
\end{abstract}

Keywords Emulsion $\cdot$ Isometric contractility $\cdot$ Uterus $\cdot$ Solvents $\cdot$ Tissue bath $\cdot$ Tocolytic

\section{Introduction}

The myometrium serves as a therapeutic target for labor induction and management of dysmenorrhea, preterm labor, and postpartum hemorrhage. The discovery and development of novel compounds to regulate myometrial contractility routinely utilize an ex vivo organ bath assay [1, 2]. Unfortunately, the assay requires solubilization of the test compound into a water-based, ion-balanced, biological buffer, which is largely dependent on the compound's polar character. An estimated $40 \%$ of approved drugs and

Jennifer Herington

Jennifer.1.herington@vumc.org

1 Department of Pediatrics, Division of Neonatology, Vanderbilt University Medical Center, 2215B Garland Ave, 1125 Light Hall, Nashville, TN 37232, USA

2 Department of Pharmacology, Vanderbilt University, Nashville, TN, USA nearly $90 \%$ of all drugs within the developmental pipeline consist of poorly soluble compounds [3].

Currently, there are several methods for overcoming poor compound solubility, including the modification of physicochemical properties of compounds through suitable salt forms, drug delivery systems (solvents, cosolvents, surfactants, emulsifiers, liposomes, and nanoparticles), and many others, as aptly reviewed by Kalepu and Nekkanti [3-5]. In the current study, we focused on the most common drug solubilization approaches (solvents, cosolvents, surfactants, complexions, and emulsions) to overcome poor water solubility in ex vivo organ bath contractility assays. The solubility of a compound is dependent on the polar relationship between it and the solvent [6]. Polar solvents, including ethanol (EtOH), isopropanol (IPA), methanol $(\mathrm{MeOH})$, and water, dissolve ionic and polar compounds. Semi-polar solvents, such as acetone (ACE), acetonitrile $(\mathrm{MeCN})$, dimethylacetamide (DMA), dimethylformamide (DMF), dimethyl sulfoxide (DMSO), and ethyl acetate (EtAc), can improve the miscibility of nonpolar compounds in polar solvents [4]. Typically, 
nonpolar solvents are not used in ex vivo or in vitro studies due to their high toxicity and poor water miscibility which commonly include hexanes and alkyl halides [7-10]. Surfactants contain both hydrophilic and hydrophobic groups that help solubilize water-insoluble compounds through the formation of micelles [11]. Emulsions are mixtures of two liquid phases, usually oil and water, in which one is dispersed into the other using an emulsifier, such as a surfactant, to increase its kinetic stability [11].

Currently, most reported studies using ex vivo organ baths to study myometrial contractility utilize water, DMSO, or $\mathrm{EtOH}$ to dissolve their compound(s) of interest $[2,12,13]$. To this end, we were not able to identify prior reports that examined the ability of other solvents and approaches to solubilize compounds in the ex vivo myometrial contractility organ bath assay without affecting myometrial contractility. Therefore, the goal of this study was to evaluate the effect of other solvents, cosolvents, detergents, complexions, and emulsions in ex vivo uterine contractility assays to provide the field with drug delivery options for overcoming issues in drug solubility.

\section{Materials and Methods}

\section{Reagents}

Solvents, surfactants, cosolvents, oils, and serum albumin were used from $100 \%$ stock unless otherwise noted. Acetone (A949), acetonitrile (A955), and ethyl acetate (E145) were purchased from Fisher Chemical. Isopropanol (I9516), dimethyl sulfoxide (D1435), ethanol (E7023), methanol (646377), N,N-dimethylformamide (D4551), human serum albumin (A1653), sesame oil (S3547), Cremophor EL (238470), nifedipine (N7634), Triton X-100 (T9284), and Tween 80 (P1754) were all purchased from Millipore Sigma. Polyethylene glycol 400 (B21992) and N,Ndimethylacetamide (22916) were purchased from Alfa Aesar. Lastly, vitamin E TPGS (HY-B0717) was purchased from MedChemExpress.

\section{Tissue Collection}

Experiments involving mice received prior approval from Vanderbilt University Institutional Animal Care and Use Committee and conformed to the guidelines established by the National Research Council Guide for Care and Use of Laboratory Animals. Adult (8-12 weeks of age) CD-1® IGS mice (Charles River Laboratory) were housed in 12-h light:12-h dark cycles with free access to food and water. Copulation plugs following overnight breeding defined the first day of pregnancy, with normal delivery occurring on day 19.5. Mice were euthanized on gestation day 19 with an overdose of isoflurane followed by cervical dislocation. The uterus was excised via cesarean section. After removal of fetuses, placentas, and amniotic and endometrial membranes, the myometrium was used in ex vivo organ bath contractility assays.

\section{Surfactant, Cosolvent, and Complexation Preparation}

Twofold serial dilutions $(0.03125-0.5 \%)$ of the following were made in deionized (DI) water: Triton X, Tween 80 , PEG 400, Cremophor EL, and vitamin E D- $\alpha$-tocopherol polyethylene glycol succinate. A twofold serial dilution $(0.266-4.25 \%)$ of human serum albumin was made in DI water to reflect its serum concentration [14]. A stock $0.1 \mathrm{M}$ solution of nifedipine was made in $100 \%$ ethanol. Dilutions of the cosolvents, surfactants, and the complexion were measured out to $1 \mathrm{~mL}$ each in $1.5-\mathrm{mL}$ glass vials and then heated to $37^{\circ} \mathrm{C}$ via a warm water bath. Nifedipine was added such that the final concentration in each tested ratio was $0.1 \mathrm{mM}$. Solubility of each dilution was evaluated visually for the macroscopic appearance of precipitate. The concentration capable of fully or partially solubilizing nifedipine in solution was tested in an ex vivo organ bath assay.

\section{Emulsion Preparation}

An oil in water $(\mathrm{O} / \mathrm{W})$ emulsion was prepared at a 10/70/20 ratio of oil, water, and vitamin E D- $\alpha$-tocopherol polyethylene glycol succinate (TPGS). The non-ionic surfactant TPGS was chosen as an emulsifier based on its low critical micellar concentration $(\mathrm{cmc}=0.02 \% \mathrm{w} / \mathrm{w})$, lack of toxicity, and high hydrophilic-lipophilic balance value (HLB = 13.2) [15-17]. This was deemed suitable for immediate formation of $\mathrm{O} / \mathrm{W}$ droplets and efficient dispersion of these droplets within the aqueous environment for stability throughout the experimental duration. The oil phase (sesame oil) was added to the water phase containing TPGS $(1.8 \% \mathrm{w} / \mathrm{v})$ and then emulsified using a homogenizer (VWR PowerMax AHS 200), at a fixed homogenization rate of $24,000 \mathrm{rpm}$ for 2 min to reduce droplet size by shear stress. For emulsions containing the tocolytic drug nifedipine, the drug was first dissolved in acetonitrile and added continuously to the pre-emulsified $\mathrm{O} / \mathrm{W}$ emulsion and homogenized for an additional 1 min under identical conditions. Acetonitrile was selected as the solvent based on both its low physiological effect on mouse myometrial tissue, as reported in this study, and efficacy in solubilizing nifedipine. The stability of each emulsion was evaluated visually for the macroscopic appearance of precipitate and separation of oil phase from water phase. Final concentrations of vitamin E TPGS and acetonitrile in tissue bath were $0.0013 \% \mathrm{w} / \mathrm{v}$ and $0.02 \% \mathrm{v} / \mathrm{v}$, respectively. 


\section{Ex Vivo Organ Bath Contractility Assay}

The myometrium was cut into uniform longitudinal strips $(1 \mathrm{~cm} \times 0.5 \mathrm{~cm} \times 0.1 \mathrm{~cm})$ for use in organ bath isometric contractility assays as previously described $[12,13]$. Briefly, myometrial strips were submerged into oxygenated $\left(95 \% \mathrm{O}_{2}\right.$ and $\left.5 \% \mathrm{CO}_{2}\right)$ and heated $\left(37{ }^{\circ} \mathrm{C}\right)$ Radnoti organ baths containing pH 7.4 Krebs-bicarbonate solution (KBS) $(136.7 \mathrm{mM}$ $\mathrm{NaCl}, 4.7 \mathrm{mM} \mathrm{KCl}, 2.5 \mathrm{mM} \mathrm{CaCl}_{2}, 25.2 \mathrm{mM} \mathrm{NaHCO}$, $1.5 \mathrm{mM} \mathrm{MgCl}, 1.8 \mathrm{mM} \mathrm{NaH}_{2} \mathrm{PO}_{4}$, and $15 \mathrm{mM}$ glucose per liter of deionized water). Tissue was placed under $1 g$ of tension and allowed to equilibrate for $1 \mathrm{~h}$, during which rhythmic and spontaneous contractions developed. After recording spontaneous contractility, solvents were cumulatively added every $10 \mathrm{~min}$ in twofold serial dilutions for a final concentration of $0.025-1.000 \%$. In separate experiments, the emulsion base (o/w emulsion without nifedipine), nifedipine emulsion, and nifedipine in solvent $(\mathrm{MeCN})$ were added cumulatively every $10 \mathrm{~min}$ as 10 -fold serial dilutions for a final concentration of $10 \mathrm{pM}-0.1 \mathrm{mM}$

Contractions were recorded using PowerLab/8SP and analyzed using ADInstruments LabChart 7Pro software. Contractile activity was analyzed for amplitude (average cyclic height per $10 \mathrm{~min}$ ), frequency (number of contractions per $10 \mathrm{~min}$ ), and integral (AUC relative to both baseline and period of duration $(\sim 600 \mathrm{~s})$ ). All analyses were expressed as percent change from baseline spontaneous contractility.

\section{Statistical Analysis}

The effect of solvents and emulsions on myometrial contractility utilized at least 3-5 mice (3-8 tissue strips per mouse) per group. Statistical analyses and graphic visualizations were performed with GraphPad Prism 6.0 software. Data are expressed as mean \pm SEM. Doseresponse curves were analyzed first for the preferred model: three-parameter versus four-parameter fit. The three-parameter fit was the preferred model for the solvent data, while a four-parameter log fit was preferred for emulsion data. Finally, the CRC was used to determine whether (1) one curve fits all the data sets using extra sum-of-squares $F$ test and (2) the $\mathrm{EC}_{50}$ and/or $E_{\max }$ values differ between data sets. A two-way analysis of variance followed by a post hoc Fisher's LSD test was used to determine significant differences between the $\%$ response for each concentration of a given solvent versus the control (KBS). A two-way analysis of variance followed by a post hoc Tukey test for multiple comparisons was used to determine significant $(p \leq$ 0.05 ) differences between the \% response for each concentration of emulsion base, nifedipine emulsion, and nifedipine in solvent $(\mathrm{MeCN})$.

\section{Results}

\section{Exploration of Solvent Effects on Myometrial Contractility}

The effects of common solvents used to dissolve waterinsoluble drugs were investigated on the contractility of myometrial strips from pregnant mice. Also investigated were controls, including the KBS used in the organ bath assay allowing examination of experimental duration on myometrial contractility, as well as water which is often used as the solvent for polar compounds. Representative recordings of spontaneous contractions (measured in grams of tension) prior to the cumulative additions of increasing concentrations of solvents and controls are shown in Fig. 1. After analyzing the area under the contractile curve (AUC; Fig. 2), which takes into account the contractile frequency, amplitude, and duration, we found the solvent with the least effect on myometrial contractility was EtOH, which was statistically insignificant ( $p$ $>0.05$ ) from the control KBS at every concentration (Supplemental Table 1). Furthermore, ACE and $\mathrm{MeCN}$ had moderate effects on myometrial contractility, showing statistically significant differences compared to control only at $0.5 \%$ and $1 \%$ concentration, respectively. We observed that EtAc, IPA, and DMA had the greatest inhibition on myometrial contractility reaching $\sim 30 \%$ inhibition at $0.1 \%$ (EtAc and IPA) and $0.25 \%$ (DMA), and maximum efficacy of greater than $80 \%$ inhibition.

We report that amplitude data (Fig. 3) is largely reflective of AUC data. Some of the least modulating solvents based on amplitude include $\mathrm{EtOH}$ and $\mathrm{MeCN}(\sim 50 \%$ maximum inhibition) which is congruent to AUC. However, unlike the AUC, DMSO had the weakest effect on myometrial amplitude reaching only $19 \%$ maximum inhibition. The most modulating solvents, as a function of amplitude, include EtAc, DMA, and IPA (97\%, 77\%, and $75 \%$ inhibition, respectively) similar in their effect on AUC. We observed that while some solvents significantly (supplemental Table 1) decreased the contractile AUC at the highest concentrations of solvents examined in Fig. 2, there conversely appeared to be an increase in the contractile frequency at the same percentage. To this end, $\mathrm{MeOH}$ mildly inhibited myometrial contractility with regard to AUC but displayed $\sim 50 \%$ stimulation of contraction frequency (Fig. 4). This trend is also observed for $\mathrm{EtOH}, \mathrm{MeCN}$, and $\operatorname{ACE}(21 \%, 63 \%$, and $17 \%$, respectively). Despite this finding, all other tested solvents (DMSO, DMA, DMF, EtAc, and IPA) show inhibition of contractile frequency. Like AUC and amplitude, EtAC caused the most inhibitory (98\%) effect on contractile frequency.

The majority of tested solvents began inhibiting myometrial contractions between 0.125 and $0.25 \%$ concentrations (supplemental Table 1). With this considered, Table 1 shows the myometrial relaxant effect of solvents based on 
Fig. 1 Representative contractility tracings of myometrial tissue treated with various solvents. Representative recordings of spontaneous contractility (S) prior to treatment with cumulative additions of solvents $(0.025-1.0 \%)$
KBS

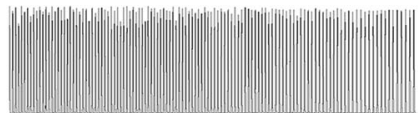

EtAc

Water

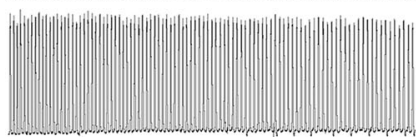

$\mathrm{EtOH}$

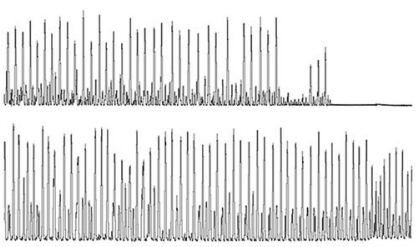

ACE

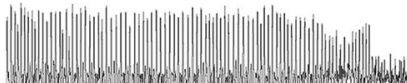

IPA

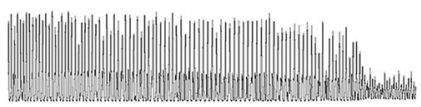

DMA

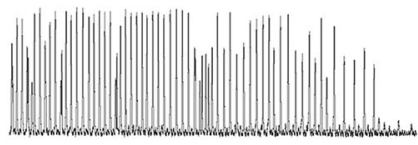

$\mathrm{MeCN}$
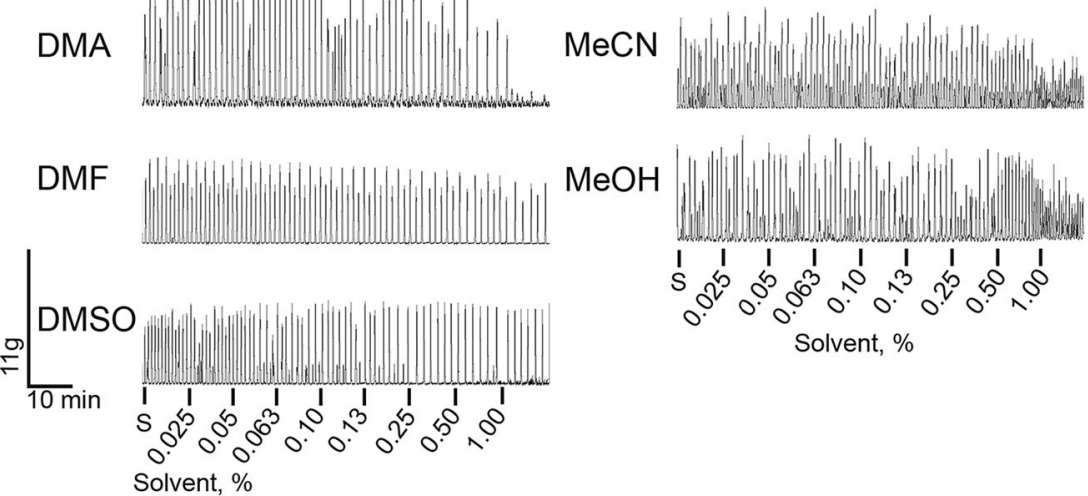

$\mathrm{MeOH}$

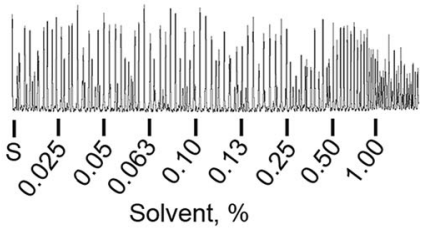

a

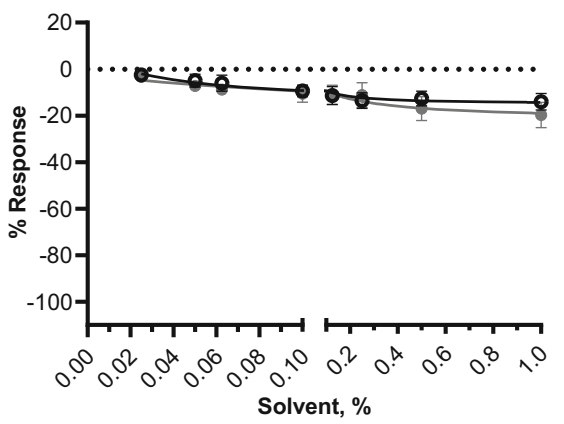

C

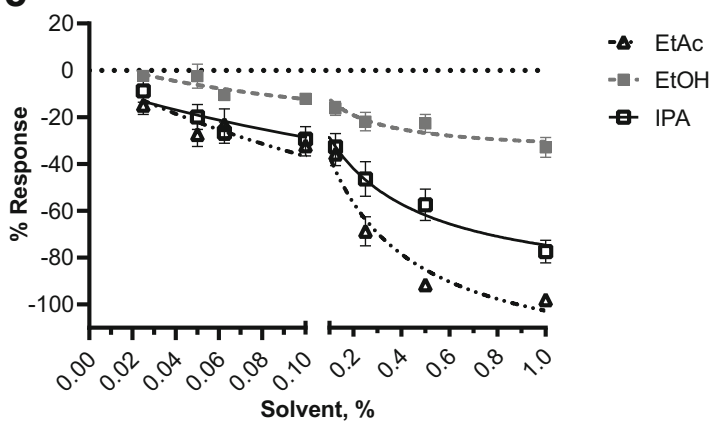

Fig. 2 Effect of solvents on ex vivo myometrial contractile area under curve. Isometric tension recordings were analyzed for integration relative to baseline (AUC normalized to the treatment duration, $\sim 600 \mathrm{~s}$ ) for each cumulative addition of solvent. Concentration-response curves were visualized using a three-parameter nonlinear fit. Data is shown as mean \pm SEM \% response relative to baseline (spontaneous contractility, S) from 3 b

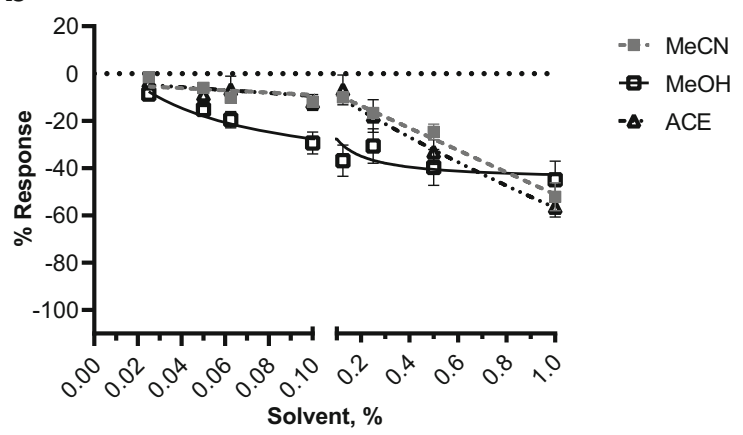

d

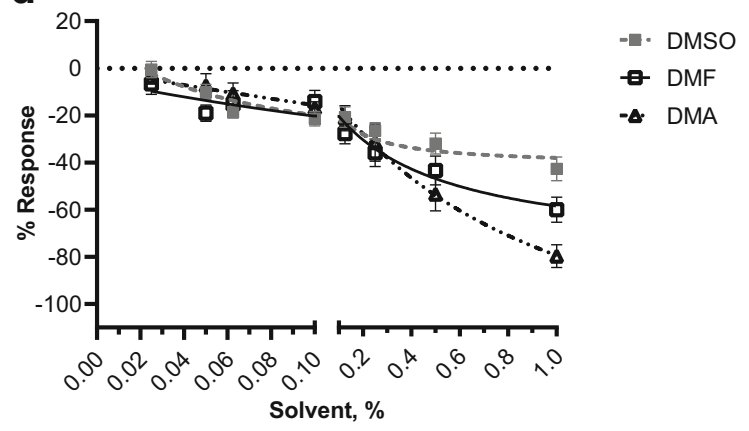

to 8 myometrial strips from $\mathrm{d} 19$ term pregnant mice $(N \geq 5)$. A two-way analysis of variance followed by a post hoc Fisher's LSD test was used to determine significant differences between the \% response for each concentration of a given solvent versus KBS and reported in supplemental Table 1 
a

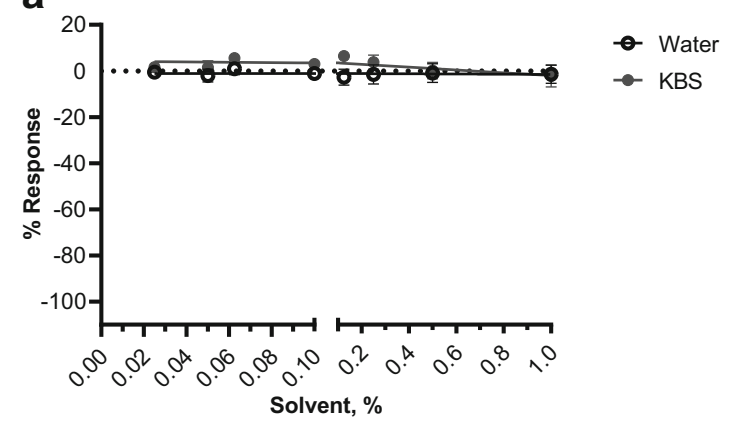

C

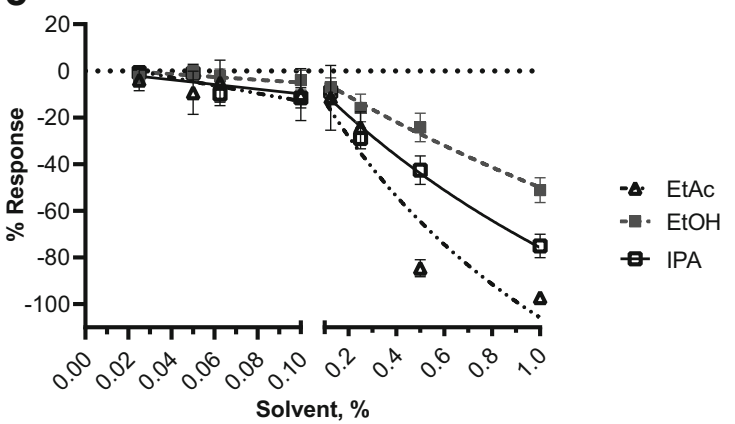

Fig. 3 Effect of solvents on ex vivo myometrial contractile amplitude. Tissue contraction recordings were analyzed for average cyclic height (contractile amplitude) for each cumulative addition of solvent. Concentration-response curves were visualized using a three-parameter nonlinear fit. Data is shown as mean \pm SEM \% response relative to
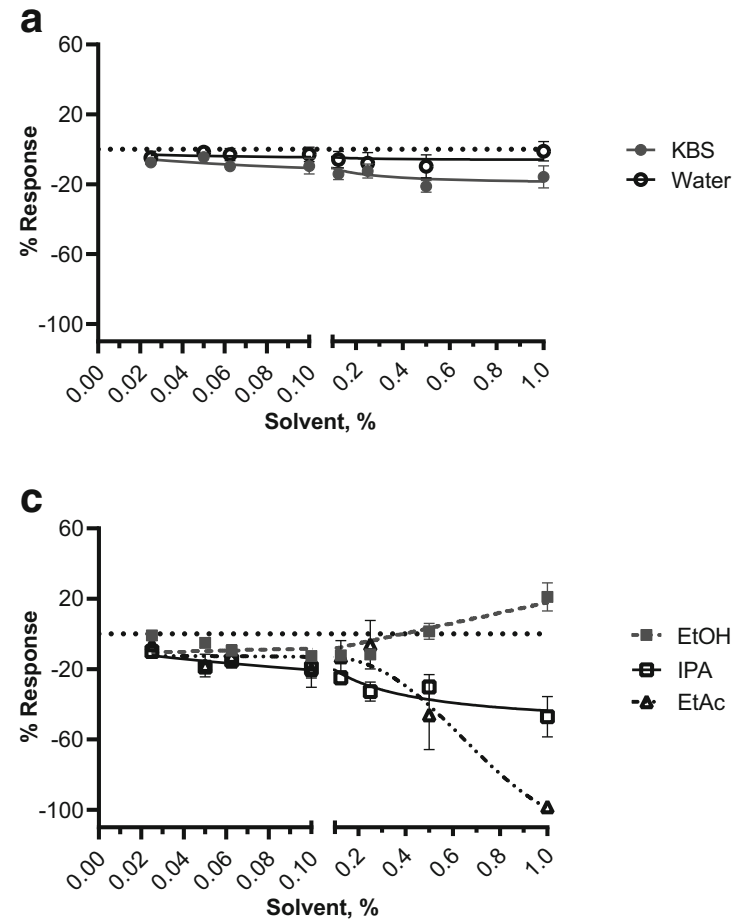

Fig. 4 Effect of solvents on ex vivo myometrial contractile frequency. Tissue contraction recordings were analyzed for relative frequency per $10 \mathrm{~min}$ for each cumulative addition of solvent. Concentration-response curves were visualized using a three-parameter nonlinear fit. Data is shown as mean \pm SEM \% response relative to baseline (spontaneous b

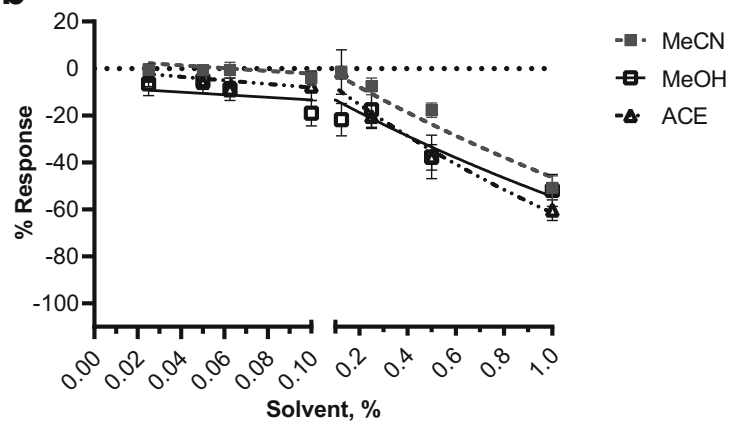

d

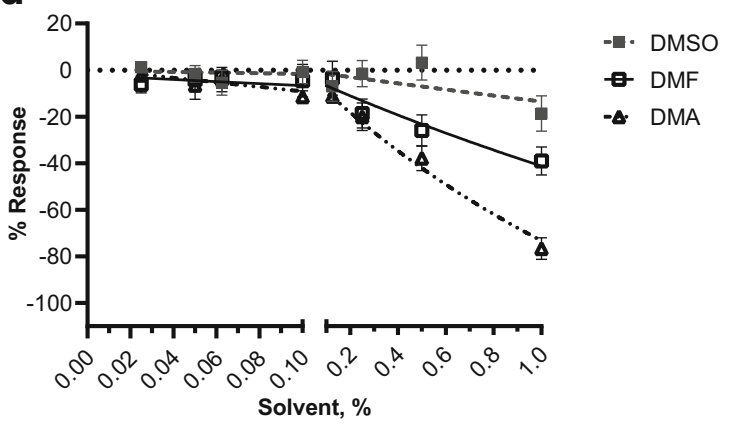

baseline (spontaneous contractility, S) from 3 to 8 myometrial strips from d19 term pregnant mice $(N \geq 5)$. A two-way analysis of variance followed by a post hoc Fisher's LSD test was used to determine significant differences between the \% response for each concentration of a given solvent versus KBS and reported in supplemental Table 1

b

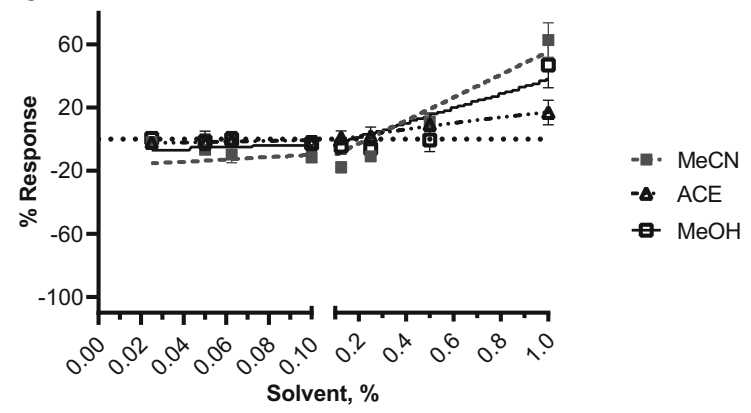

d

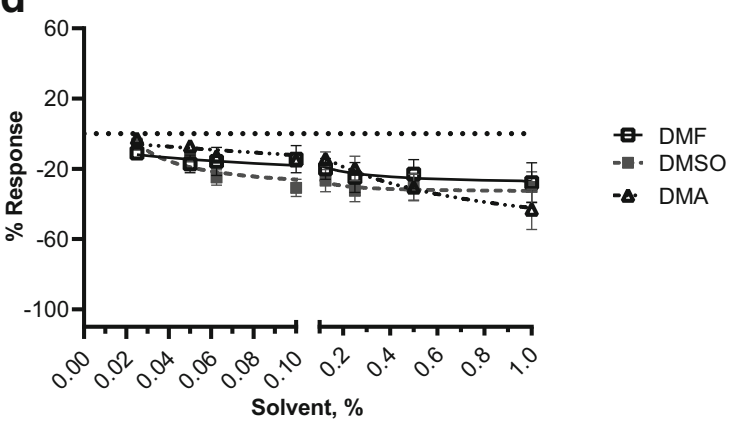

contractility, S) from 3 to 8 myometrial strips from d19 term pregnant mice $(N \geq 5)$. A two-way analysis of variance followed by a post hoc Fisher's LSD test was used to determine significant differences between the \% response for each concentration of a given solvent versus KBS and reported in supplemental Table 1 
AUC at $0.1 \%$, the concentration directly below $0.125 \%$. At the timepoint ( $\sim 2 \mathrm{~h}$ after the start of the experiment) that $0.1 \%$ of solvent was added in the organ bath assay, the control (KBS and water) treated myometrial tissues experienced $11 \%$ and $9 \%$ inhibition, respectively, in their contractile activity. Solvents with inhibitory contractile effects within the same range as these controls at the same experimental timepoint include the following: $\mathrm{MeCN}$, EtOH, and ACE. However, solvents that had a significantly greater inhibitory effect at the $0.1 \%$ addition included the following: DMSO, EtAc, IPA, and $\mathrm{MeOH}$ (Table 1).

\section{Determination of Surfactant, Cosolvent, and Complexation Effects in the Organ Bath}

The most common pharmacological approaches to solubilize poorly water-soluble compounds include surfactant addition, cosolvency, and complexation [4, 18, 19]. We explored the effects of the common surfactants Tween 80, Triton X-100, vitamin E TPGS, and Cremophor EL at concentrations of $0.03125-0.5 \% \mathrm{v} / \mathrm{v}$ in an effort to fully or partially solubilize nifedipine, a water-insoluble compound that is clinically utilized as a tocolytic. Tween 80 was the only surfactant to fully solubilize the compound visually at the highest tested concentration $(0.5 \% \mathrm{v} / \mathrm{v})$, while the other surfactants studied only partially solubilized the drug. The commonly used cosolvent PEG $400(0.5 \% \mathrm{v} / \mathrm{v})$ and a water-soluble complexation of human serum albumin $(4.25 \% \mathrm{w} / \mathrm{v})$ were also tested in the organ bath at a maximum concentration required to fully or partially solubilize nifedipine visually. Polyethylene glycol 400 completely lacked the ability to solubilize nifedipine at

Table 1 Effect of solvents and controls on myometrial contractility at $0.1 \%$ concentration.

The table lists the mean \pm SD AUC relative to baseline at $0.1 \%$ concentration of the solvents referenced in Fig. 2

\begin{tabular}{ll}
\hline & \% Response \\
\hline Controls & \\
KBS & $-10.55 \pm 10.51$ \\
Water & $-9.44 \pm 10.14$ \\
Solvents & \\
MeCN & $-11.92 \pm 7.53$ \\
EtOH & $-12.15 \pm 5.18$ \\
ACE & $-12.24 \pm 10.98$ \\
DMF & $-14.17 \pm 15.94$ \\
DMA & $-16.87 \pm 8.90$ \\
DMSO & $-21.40 \pm 10.63$ \\
IPA & $-29.40 \pm 18.62$ \\
MeOH & $-29.44 \pm 15.51$ \\
EtAc & $-32.21 \pm 12.29$ \\
Emulsion & \\
MeCN Vit E-TPGS & $-15.74 \pm 13.94$
\end{tabular}

Values shown are mean \pm SD for contractile AUC at $0.1 \%$ solvent even the highest concentration tested $(0.5 \% \mathrm{v} / \mathrm{v})$. Unfortunately, since the ex vivo organ bath contractility assay involves constant aeration with $95 \% \mathrm{O}_{2} / 5 \% \mathrm{CO}_{2}$, the surfactants and albumin in KBS cause extensive bubbling (Supplementary Figure 1) and thus lack the merit for further exploration.

\section{Investigation of Emulsion Effects on Myometrial Contractility}

An oil-in-water emulsion was prepared to examine whether this formulation strategy might be useful for ex vivo organ bath contractility assays. As a proof-of-principle, we also tested emulsions containing nifedipine and compared the drug's efficacy and potency when administered as an emulsion versus freely dissolved in the solvent MeCN. Representative recordings of spontaneous contractions prior to the cumulative additions of increasing concentrations of nifedipine (emulsion and in $\mathrm{MeCN}$ ) and control (emulsion base) are shown in Fig. 5a. We found that the myometrial tissue strips treated with increasing concentrations of the emulsion base (Fig. 5b-d) elicited a similar degree of inhibition at $\sim 2 \mathrm{~h}$ after the start of the experiment as the controls KBS and water (Figs. 2, 3, and 4). After increasing concentrations of nifedipine were added to the organ bath, either as an emulsion or in a solvent, we observed similar inhibition (Fig. 5b-d) and no significant difference between the $\mathrm{EC}_{50}$ and $E_{\max }$ (Fig. $5 \mathrm{~b} ; p=0.87$ ). As seen in Fig. $5 \mathrm{c}$ and $\mathrm{d}$, the amplitude and frequency of emulsion data are congruent with that of AUC.

\section{Discussion}

Early drug discovery, involving in vitro and ex vivo experiments, largely relies on aqueous-based assays. Ex vivo organ bath contractility assays using myometrial tissue remain an important screening tool for the discovery and development of effective, novel tocolytics and uterotonics [2, 12, 20-22]. It is crucial for the investigational compound to be in a homogenous solution within the organ bath buffer for accurate determination of a compound's efficacy and potency. Currently, there are several solubilization methods; however, determining which technique to use depends largely upon its utility within a specific assay, taking into consideration tissue and cell effects, buffer composition, and test compound polarity [3, 23-27]. Many organ bath studies on a variety of muscle types have used various different solvents (DMSO, EtOH, $\mathrm{MeOH}$, EtAc) to solubilize test compounds [28-32]. However, we were not able to identify prior reports that examined the ability of various solvents and solubilization approaches that adequately solubilize compounds in the ex vivo myometrial contractility organ bath assay without affecting myometrial contractility. Therefore, the present study 
a
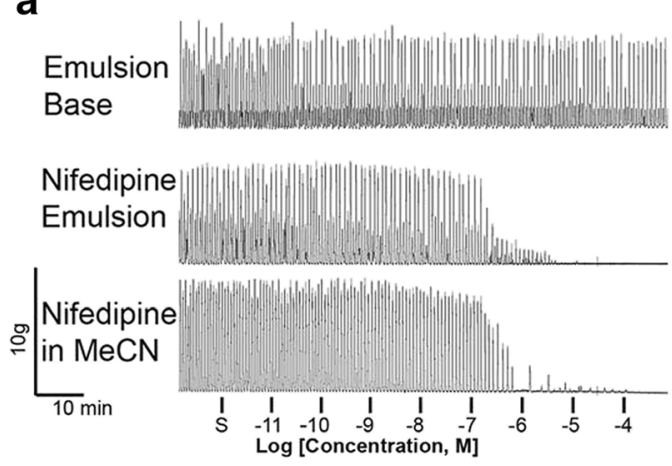

C

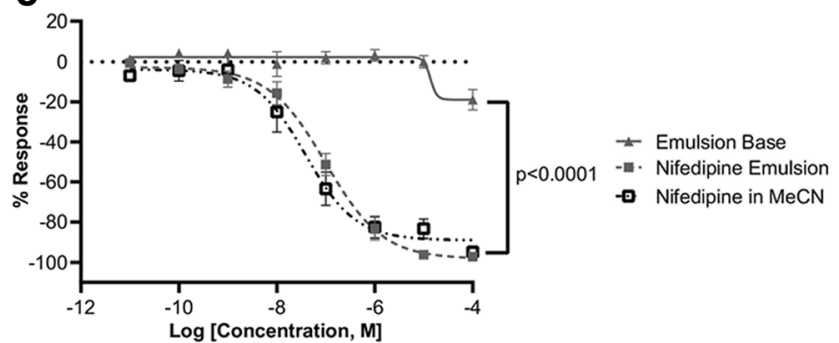

Fig. 5 Utility of an emulsion as a formulation for ex vivo organ bath contractility assay. a Representative recordings of spontaneous contractility prior to cumulative treatment $(10 \mathrm{pM}-0.1 \mathrm{mM})$ every 10 min with either base emulsion, nifedipine (NIF) emulsion, or NIF in $\mathrm{MeCN}$. Isometric tension recordings were analyzed for AUC (b), amplitude (c), and frequency (d). All concentration-response curves, including

provides insight into the effect of common solvents, surfactants, cosolvents, and emulsions on myometrial contractility in order to identify delivery methods for water-insoluble compounds with a minimal effect on myometrial contractility.

The present study indicates that EtAc, IPA, and $\mathrm{MeOH}$ dampen ex vivo mouse myometrial contractions at experimentally relevant concentrations, based on AUC and change in amplitude, compared to water and KBS controls. The most modulatory solvent reported in this study was EtAc, showing a nearly $100 \%$ inhibition of contractility at $0.5 \% \mathrm{v} / \mathrm{v}$ solvent. In contrast, the least modulatory solvent at the highest tested concentration was $\mathrm{EtOH}$, having $\sim 30 \%$ inhibition. Considering the inhibitory effect of the majority of studied solvents at concentrations greater than $0.125 \%$, it appears that $0.1 \%$ serves as an appropriate threshold for solvent percentage within the myometrial contractility organ bath assay. The present study found that $\mathrm{ACE}, \mathrm{EtOH}$, and $\mathrm{MeCN}$ are the most desirable solvents for water-insoluble compounds. Conversely, DMSO, EtAc, IPA, and $\mathrm{MeOH}$ had an inhibitory effect statistically greater than the control solvents water and KBS and thus would not be desirable solvents for compounds in the myometrial organ bath assay. Amplitude data is congruent with AUC contractile data. However, we observed that $\mathrm{MeOH}, \mathrm{MeCN}, \mathrm{EtOH}$, and $\mathrm{ACE}$ induce contractile frequency while inhibiting amplitude, which is supported by other studies [30].

Ethanol is a known uterine-relaxant. In fact, prior to its discontinued use due to detrimental fetal effects, ethanol was

\section{b}
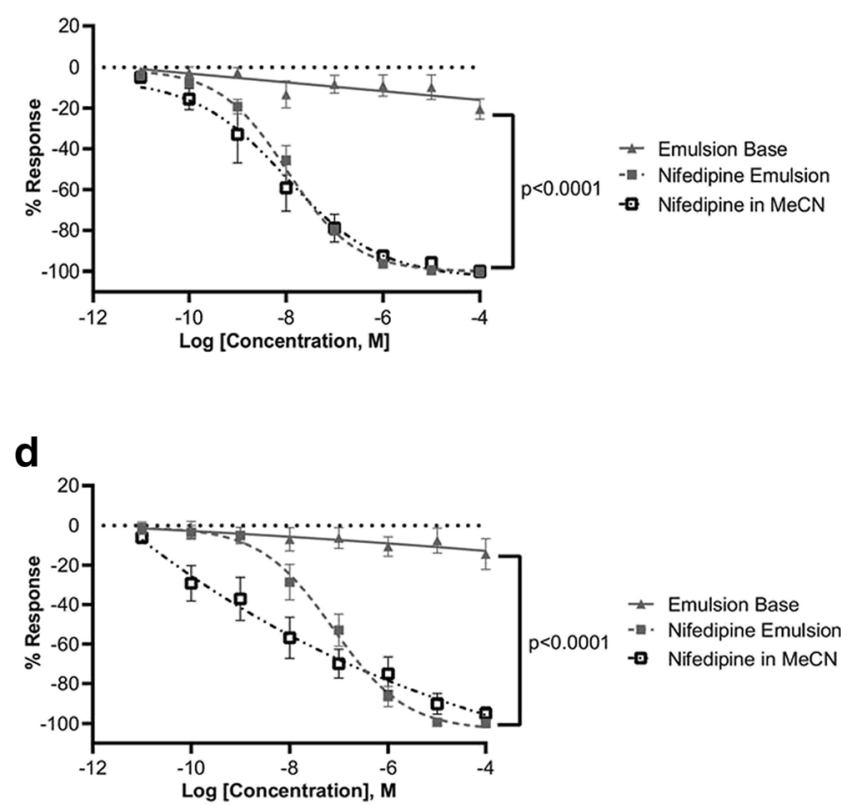

AUC, amplitude, and frequency, were visualized using a four-parameter nonlinear $\log$ fit. Data is shown as mean $\pm \mathrm{SEM} \%$ response relative to baseline (spontaneous contractility, S) from 3 to 4 myometrial strips from d19 term pregnant mice $(N \geq 3)$. Significant $(p<0.0001)$ difference between each fit line is indicated

utilized clinically as a tocolytic agent [33-36]. However, the concentration required to achieve tocolytic benefit was 9.5 $10 \%[35,36]$. Low-dose ethanol was found to be no more effective than placebo $[37,38]$ as a tocolytic to manage preterm labor. Similarly, the data presented here shows that EtOH causes no significant tocolytic effect in the organ bath assay for concentrations tested $(\leq 1 \%)$, as analyzed by AUC.

Surfactants, cosolvents, and complexation agents are routinely added as solubilizing agents for in vitro assays $[18,19$, 39]. For highly hydrophobic compounds, the concentration of solvent or surfactant required for solubilization can be detrimental to tissue activity or affect pharmacological assays [28, $39,40]$. Therefore, we tested the minimum concentration of surfactants, a cosolvent, and serum albumin required to solubilize nifedipine, a current tocolytic drug that is poorly soluble in water. As mentioned in the results, the bubbling experienced in the organ baths at the required concentrations discouraged further exploration. There have been reports of using perfluorodecalin to stabilize bubbles caused by the use of pulmonary surfactants, without affecting the assay system [41]. However, this approach to reduce bubbling in myometrial organ bath has not been reported, and the effect on myometrial contractility has yet to be established.

Emulsion-based delivery systems are frequently used as a formulation strategy for compounds with poor aqueous solubility in later stages of development [5, 27, 42-44]. In the present study, we investigated the potential of emulsion- 
based formulations as a delivery system for insoluble compounds in organ bath experiments. There are published reports on emulsions tested in organ baths on rat aortic tissue [45, 46]; however, this is the first report of emulsions being used as a solubilization method for poorly soluble compounds in an organ bath assay using mouse myometrial tissue. Importantly, we found that the base emulsion without drug was not different to that of water or KBS. Furthermore, it is seen that the emulsion of nifedipine in comparison to the freely solubilized nifedipine (in $\mathrm{MeCN}$ ) is also not statistically significant, thus indicating that nifedipine in an emulsion is able to penetrate the tissue to elicit similar potency and efficacy. Here, we show that a base emulsion is aptly absorbed by tissue with little effect on normal function. This is an important note because solubility, though an obstacle, is not the goal in drug development. The ability of the tissue to absorb the drug, regardless of formulation, is more important. This suggests that emulsions may be effective delivery systems for poorly, or completely, water-insoluble compounds in this assay.

Isolated tissue bath contractility experiments are commonly used in pharmacological research on various tissues [2, 47-51]. While our study explores the effects of different solvents and emulsions on myometrial tissue, the known effect of each solvent may not be translatable to all tissue types explored in organ bath studies. Thus, there is a need to establish solvent effects for each tissue tested, similar to that of tissue selectivity for novel compounds, in organ baths [52-57]. Combinatorial solvent systems have also been used to maintain solubility for nonpolar compounds, such as 2:1 DMSO:EtOH [21]. Combination of solvents could alter myometrial tissue differently compared to individual solvents. Future studies that explore solvent combinations, and/or combinations with other additives mentioned here in tissue contractility assays, could benefit from the data presented in this study. Furthermore, the study described here was performed on mouse tissue, and therefore, the effects described here should be used as a starting point to assess solvents and drug solubility in studies on human myometrial strips, another tissue commonly used to assess the tocolytic or uterotonic efficacy and potency of compounds. Moreover, the emulsions tested here were done as a proof-of-concept to establish the possibility of usage; however, different compounds may require tailored emulsion formulations and longer stability studies depending on experimental parameters. Our emulsion was visually tested to be stable for 3-4 h, longer than the experiment duration using mouse myometrial tissue.

Our data provides a platform for the expanded understanding of the use of solvents and emulsion as drug delivery options in ex vivo myometrial organ bath systems. The work indicates that EtAc, IPA, and DMA have strong, contractile inhibitory effects, while $\mathrm{ACE}, \mathrm{EtOH}$, and $\mathrm{MeCN}$ have minimal effects on mouse myometrial tissue in organ bath assays at concentrations $\leq 0.1 \%$. Furthermore, emulsions merit further exploration for their ability to maintain stable mixtures within this assay allowing compounds' physiological effects to be explored.

Supplementary Information The online version contains supplementary material available at https://doi.org/10.1007/s43032-021-00576-5.

Code Availability Not applicable

Funding This project was supported by research funds from the Eunice Kennedy Shriver National Institute of Child Health and Human Development grant numbers HD088830 and HD098213 (JLH).

Data Availability The data and material from this study are readily available from the senior author, Jennifer Herington.

\section{Declarations}

Ethics Approval Experiments involving mice received prior approval (protocol no. M1600262-01) from Vanderbilt University Institutional Animal Care and Use Committee and conformed to the guidelines established by the National Research Council Guide for Care and Use of Laboratory Animals.

Consent to Participate Not applicable.

Consent for Publication All authors consent to the publication of this study.

Conflict of Interest The authors declare no competing interests.

Open Access This article is licensed under a Creative Commons Attribution 4.0 International License, which permits use, sharing, adaptation, distribution and reproduction in any medium or format, as long as you give appropriate credit to the original author(s) and the source, provide a link to the Creative Commons licence, and indicate if changes were made. The images or other third party material in this article are included in the article's Creative Commons licence, unless indicated otherwise in a credit line to the material. If material is not included in the article's Creative Commons licence and your intended use is not permitted by statutory regulation or exceeds the permitted use, you will need to obtain permission directly from the copyright holder. To view a copy of this licence, visit http://creativecommons.org/licenses/by/4.0/.

\section{References}

1. Siricilla S, Iwueke CC, Herington JL. Drug discovery strategies for the identification of novel regulators of uterine contractility. Curr Opin Physiol. 2020;13:71-86.

2. Arrowsmith $\mathrm{S}$, et al. Contractility measurements of human uterine smooth muscle to aid drug development. J Vis Exp. 2018;131.

3. Kalepu S, Nekkanti V. Insoluble drug delivery strategies: review of recent advances and business prospects. Acta Pharm Sin B. 2015;5(5):442-53. 
4. Nayak AK, Panigrahi PP. Solubility enhancement of etoricoxib by cosolvency approach. ISRN Phys Chem. 2012;2012:820653.

5. Li Y, le Maux S, Xiao H, McClements DJ. Emulsion-based delivery systems for tributyrin, a potential colon cancer preventative agent. J Agric Food Chem. 2009;57(19):9243-9.

6. Martin A, Wu PL, Liron Z, Cohen S. Dependence of solute solubility parameters on solvent polarity. J Pharm Sci. 1985;74(6):63842.

7. Browning E. Toxic solvents: a review. Br J Ind Med. 1959;16(1): 23-39.

8. Kulkarni S. Alkyl Halides. In: Philip Wexler, editor. Encyclopedia of toxicology (third edition). Academic Press; 2014. p. 144145. https://doi.org/10.1016/B978-0-12-386454-3.00229-3.

9. Medicine Io. Gulf War and health: volume 2: insecticides and solvents. Washington, DC: The National Academies Press; 2003. p. 616.

10. Raje RR. In vitro toxicity of n-hexane and 2,5-hexanedione using isolated perfused rabbit heart. J Toxicol Environ Health. 1983;11(46):879-84

11. Jaiswal M, Dudhe R, Sharma PK. Nanoemulsion: an advanced mode of drug delivery system. 3 Biotech. 2015;5(2):123-7.

12. Herington JL, O'Brien C, Robuck MF, Lei W, Brown N, Slaughter $\mathrm{JC}$, et al. Prostaglandin-endoperoxide synthase 1 mediates the timing of parturition in mice despite unhindered uterine contractility. Endocrinology. 2018;159(1):490-505.

13. Herington JL, Swale DR, Brown N, Shelton EL, Choi H, Williams $\mathrm{CH}$, et al. High-throughput screening of myometrial calciummobilization to identify modulators of uterine contractility. PLoS One. 2015;10(11):e0143243.

14. Theodore Peters J. All about albumin, biochemistry, genetics and medical applications. San Diego: Academic Press; 1996. p. 432.

15. Yang C, Wu T, Qi Y, Zhang Z. Recent advances in the application of vitamin E TPGS for drug delivery. Theranostics. 2018;8(2):464 85.

16. Zhang Z, Tan S, Feng SS. Vitamin E TPGS as a molecular biomaterial for drug delivery. Biomaterials. 2012;33(19):4889-906.

17. Zhang Z, Feng S-S. Nanoparticles of poly(lactide)/vitamin E TPGS copolymer for cancer chemotherapy: synthesis, formulation, characterization and in vitro drug release. Biomaterials. 2006;27(2): 262-70.

18. Loftsson T, Stefansson E. Cyclodextrins and topical drug delivery to the anterior and posterior segments of the eye. Int J Pharm. 2017;531(2):413-23.

19. Mouhamad Khoder HA, ElShaer A, Karam A, Najlah M, Alany RG. Efficient approach to enhance drug solubility by particle engineering of bovine serum albumin. Int J Pharm. 2016;515(1-2):740 8.

20. Kimizuka M, Tokinaga Y, Azumaguchi R, Hamada K, Kazuma S, Yamakage M. Effects of anesthetic agents on contractions of the pregnant rat myometrium in vivo and in vitro. J Anesth. 2021;6880. https://doi.org/10.1007/s00540-020-02866-9.

21. Fernandez-Martinez E, et al. Inhibition of uterine contractility by thalidomide analogs via phosphodiesterase-4 inhibition and calcium entry blockade. Molecules. 2016;21(10):1332.

22. Modzelewska B, Jóźwik M, Jóźwik M, Sulkowski S, PedzińskaBetiuk A, Kleszczewski T, et al. Altered uterine contractility in response to beta-adrenoceptor agonists in ovarian cancer. $\mathrm{J}$ Physiol Sci. 2017;67(6):711-22.

23. Williams HD, Trevaskis NL, Charman SA, Shanker RM, Charman WN, Pouton CW, et al. Strategies to address low drug solubility in discovery and development. Pharmacol Rev. 2013;65(1):315-499.

24. Bodor N. Techniques of solubilization of drugs. (Drugs and the Pharmaceutical Sciences Series, Vol 12.). J Pharm Sci. 1984;73(2):288. https://doi.org/10.1002/jps.2600730245.

25. Basavaraj S, Betageri GV. Can formulation and drug delivery reduce attrition during drug discovery and development-review of feasibility, benefits and challenges. Acta Pharm Sin B. 2014;4(1): 3-17.

26. Aravindhan Ganesan KB. Solubility: a speed-breaker on the drug discovery highway. MOJ Bioequiv Availab. 2017;3(3):56-8.

27. Gupta S, Kesarla R, Omri A. Formulation strategies to improve the bioavailability of poorly absorbed drugs with special emphasis on self-emulsifying systems. ISRN Pharmaceut. 2013;2013:848043.

28. Braak K, Frey HH. Effects of solvents and detergents on the contractions of isolated smooth muscle preparations. J Pharm Pharmacol. 1990;42(12):837-41.

29. Levin RM, Danek M, Whitbeck C, Haugaard N. Effect of ethanol on the response of the rat urinary bladder to in vitro ischemia: protective effect of alpha-lipoic acid. Mol Cell Biochem. 2005;271(1-2):133-8.

30. Malysz J, Afeli SAY, Provence A, Petkov GV. Ethanol-mediated relaxation of guinea pig urinary bladder smooth muscle: involvement of BK and L-type Ca2+ channels. Am J Phys Cell Phys. 2014;306(1):C45-58.

31. Verleden GM, Pype JL, Demedts MM. Ketotifen modulates noncholinergic contraction in guinea pig airways in vitro by a prejunctional nonhistamine receptor. J Allergy Clin Immunol. 1994;94(2 Pt 1):207-14.

32. Lozano-Cuenca J, Lopez-Canales OA, Aguilar-Carrasco JC, Villagrana-Zesati JR, Lopez-Mayorga RM, Castillo-Henkel EF, Lopez-Canales JS. Pharmacological study of the mechanisms involved in the vasodilator effect produced by the acute application of triiodothyronine to rat aortic rings. Braz J Med Biol Res. 2016. https://doi.org/10.1590/1414-431X20165304.

33. Gyetvai K, et al. Tocolytics for preterm labor: a systematic review. Obstet Gynecol. 1999;94(5, Part 2):869-77.

34. Keirse MJ. The history of tocolysis. Bjog. 2003;110(Suppl 20):94 7.

35. Fuchs F, Fuchs AR, Poblete VF Jr, Risk A. Effect of alcohol on threatened premature labor. Am J Obstet Gynecol. 1967;99(5):62737

36. Lauersen NH, Merkatz IR, Tejani N, Wilson KH, Roberson A, Mann LI, et al. Inhibition of premature labor: a multicenter comparison of ritodrine and ethanol. Am J Obstet Gynecol. 1977;127(8):837-45.

37. Berkman ND, Thorp JM Jr, Lohr KN, Carey TS, Hartmann KE, Gavin NI, et al. Tocolytic treatment for the management of preterm labor: a review of the evidence. Am J Obstet Gynecol. 2003;188(6): 1648-59.

38. Horiguchi T, Suzuki K, Comas-Urrutia AC, Mueller-Heubach E, Boyer-Milic AM, Baratz RA, et al. Effect of ethanol upon uterine activity and fetal acid-base state of the rhesus monkey. Am J Obstet Gynecol. 1971;109(6):910-7.

39. Randall K, Cheng SW, Kotchevar AT. Evaluation of surfactants as solubilizing agents in microsomal metabolism reactions with lipophilic substrates. In Vitro Cell Dev Biol Anim. 2011;47(9):631-9.

40. Maes J, Verlooy L, Buenafe OE, de Witte PAM, Esguerra CV, Crawford AD. Evaluation of 14 organic solvents and carriers for screening applications in zebrafish embryos and larvae. PLoS One. 2012;7(10):e43850.

41. Koetzler R, Saifeddine M, Yu Z, Schürch FS, Hollenberg MD, Green FHY. Surfactant as an airway smooth muscle relaxant. Am J Respir Cell Mol Biol. 2006;34(5):609-15.

42. Wadhwa J, Nair A, Kumria R. Emulsion forming drug delivery system for lipophilic drugs. Acta Pol Pharm. 2012;69(2):179-91.

43. Bouchemal K, Briançon S, Perrier E, Fessi H. Nano-emulsion formulation using spontaneous emulsification: solvent, oil and surfactant optimisation. Int J Pharm. 2004;280(1-2):241-51.

44. Walker S, Tailor SAN, Lee M, Louie L, Louie M, Simor AE. Amphotericin B in lipid emulsion: stability, compatibility, and in vitro antifungal activity. Antimicrob Agents Chemother. 1998;42(4):762-6. 
45. Ok S-H, Lee SH, Kim JY, Kim HJ, Bae SI, Hwang Y, et al. Lipid emulsion inhibits the vasodilation induced by a toxic dose of amlodipine in isolated rat aortae. Int J Med Sci. 2019;16(12): 1621-30.

46. Guo H, Zhang HF, Xu WQ, du Q, Zhao J, Ren LM. Differential effects of short- and long-term bupivacaine treatment on $\alpha 1$ adrenoceptor-mediated contraction of isolated rat aorta rings and the reversal effect of lipid emulsion. Acta Pharmacol Sin. 2015;36(8):976-86.

47. Peddireddy MKR. In vitro evaluation techniques for gastrointestinal motility. Indian J Pharmaceut Educ Res. 2011;45:184-91.

48. Pozzoli C, Poli E. Assessment of gastrointestinal motility using three different assays in vitro. Current Protoc Toxicol. 2010;46(1):21.8.1-21.8.33.

49. Sutter MC. The pharmacology of isolated veins. Br J Pharmacol Chemother. 1965;24:742-51.

50. Kullmann FA, et al. Bladder smooth muscle strip contractility as a method to evaluate lower urinary tract pharmacology. J Vis Exp. 2014;90:e51807.

51. Jespersen B, et al. Measurement of smooth muscle function in the isolated tissue bath-applications to pharmacology research. $\mathrm{J}$ Vis Exp. 2015;95:52324.

52. Kukovetz WR, et al. Pharmacological properties of fendiline in cardiac and smooth muscle (author's transl). Arzneimittelforschung. 1976;26(7):1321-30.
53. Hooker CS, Calkins PJ, Fleisch JH. On the measurement of vascular and respiratory smooth muscle responses in vitro. Blood Vessels. 1977;14(1):1-11.

54. Ek A, Alm P, Andersson KE, Persson CGA. Adrenergic and cholinergic nerves of the human urethra and urinary bladder. A histochemical study. Acta Physiol Scand. 1977;99(3):345-52.

55. Abdur Rahman HM, Ahmed K, Rasool MF, Imran I. Pharmacological evaluation of smooth muscle relaxant and cardiac-modulation potential of phyla nodiflora in ex-vivo and in-vivo experiments. Asian Pac J Trop Med. 2017;10(12):114653.

56. Rahman HMA, Rasool MF, Imran I. Pharmacological studies pertaining to smooth muscle relaxant, platelet aggregation inhibitory and hypotensive effects of Ailanthus altissima. Evid Based Complement Alternat Med. 2019;2019:1871696.

57. Janbaz K, Jan A, Qadir MI, Gilani AH. Spasmolytic, bronchodilator and vasorelaxant activity of methanolic extract of Tephrosia purpurea. Acta Pol Pharm. 2013;70:261-9.

Publisher's Note Springer Nature remains neutral with regard to jurisdictional claims in published maps and institutional affiliations. 